\title{
High intralocus variability and interlocus recombination promote immunological diversity in a minimal major histocompatibility system
}

\author{
Anthony B Wilson ${ }^{1,25^{*}}$, Camilla M Whittington ${ }^{1,3}$ and Angela Bahr ${ }^{1,4}$
}

\begin{abstract}
Background: The genes of the major histocompatibility complex (MHC/MH) have attracted considerable scientific interest due to their exceptional levels of variability and important function as part of the adaptive immune system Despite a large number of studies on MH class II diversity of both model and non-model organisms, most research has focused on patterns of genetic variability at individual loci, failing to capture the functional diversity of the biologically active dimeric molecule. Here, we take a systematic approach to the study of $\mathrm{MH}$ variation, analyzing patterns of genetic variation at $\mathrm{MH}$ class $\| \mathrm{a}$ and $\| \beta$ loci of the seahorse, which together form the immunologically active peptide binding cleft of the MH class II molecule.

Results: The seahorse carries a minimal class II system, consisting of single copies of both MH class Ila and II, which are physically linked and inherited in a Mendelian fashion. Both genes are ubiquitously expressed and detectible in the brood pouch of male seahorses throughout pregnancy. Genetic variability of the two genes is high, dominated by non-synonymous variation concentrated in their peptide-binding regions. Coding variation outside these regions is negligible, a pattern thought to be driven by intra- and interlocus recombination. Despite the tight physical linkage of $\mathrm{MH} \| \mathrm{la}$ and $\| \beta$ loci, recombination has produced novel composite alleles, increasing functional diversity at sites responsible for antigen recognition.

Conclusions: Antigen recognition by the adaptive immune system of the seahorse is enhanced by high variability at both MH class $\|$ a and $\| \beta$ loci. Strong positive selection on sites involved in pathogen recognition, coupled with high levels of intra- and interlocus recombination, produce a patchwork pattern of genetic variation driven by genetic hitchhiking. Studies focusing on variation at individual MH loci may unintentionally overlook an important component of ecologically relevant variation.
\end{abstract}

Keywords: Gene conversion, Major histocompatibility complex, Natural selection, Recombination, Sexual selection, Syngnathidae

\section{Background}

The genes of the major histocompatibility complex (MHC) are an essential component of the adaptive immune system, and among the most variable loci in the vertebrate genome [1]. Classical major histocompatibility $(\mathrm{MH})$ class I and II genes are responsible for the recognition of foreign

\footnotetext{
* Correspondence: twilson@brooklyn.cuny.edu

${ }^{1}$ Institute of Evolutionary Biology and Environmental Studies, University of Zurich, Winterthurerstrasse 190, 8057 Zurich, Switzerland

${ }^{2}$ Department of Biology, Brooklyn College and The Graduate Center, City University of New York, 2900 Bedford Avenue, Brooklyn, New York 11210, USA

Full list of author information is available at the end of the article
}

antigens [2,3], and the peptide-binding regions (PBR) of these genes represent some of the most striking examples of balancing selection [4]. MH class II molecules are protein dimers produced from the combination of class II $\alpha$ and II $\beta$ genes, which together form the immunologicallyactive peptide-binding cleft involved in the recognition of extracellular pathogens [5]. The high variability of the peptide-binding regions of these two genes ensures that hosts can recognize a broad spectrum of pathogens, and these regions are consequently subject to strong positive selection.

Despite the fact that antigen recognition by $\mathrm{MH}$ class II is determined by a protein complex including both II $\alpha$ 
and II $\beta$ molecules, the majority of studies have focused on only a single component of this complex (typically $\mathrm{MH}$ II $\beta)$, neglecting the fact that antigen discrimination is likely to be realized via unique permutations of $\mathrm{MH}$ II $\alpha$ and II $\beta$ alleles in the host. This gene-centric approach to the analysis of $\mathrm{MH}$ diversity has been premised on the assumption that allelic variability at class II $\alpha$ loci is negligible relative to that found at class II $\beta$ genes. While genetic variability of $\mathrm{MH}$ class II $\alpha$ is comparatively low in human populations [6], this hypothesis has not been well investigated in other vertebrate taxa.

The majority of vertebrates carry multiple copies of classical MH loci [7], complicating efforts to study intraand interlocus allelic variability in natural populations. In contrast to the tightly linked structure of major histocompatibility genes in mammals, $\mathrm{MH}$ genes are unlinked in teleost fishes, and members of this group show considerable variation in the number and structure of $\mathrm{MH}$ class I and II loci [8], providing a powerful comparative model for the study of evolutionary immunology. A recent study comparing patterns of allelic variability of $\mathrm{MH}$ II $\alpha$ and II $\beta$ loci in salmonid fishes with a minimal $\mathrm{MH}$ class II system found high levels of genetic variation at both loci, diversity that could serve to maintain the efficiency of pathogen recognition despite the absence of additional gene copies [9]. Unfortunately, given the relative paucity of studies directly investigating allelic variability of both components of the MH II molecule, it remains unclear whether the high genetic diversity found at both loci in salmonids is a lineage-specific phenomenon associated with their minimal MH system, or whether it reflects a broader pattern relevant for understanding MH class II function in other taxa.

Seahorses (Hippocampus spp.) are a genus of marine fishes well known for their unique form of reproduction, male pregnancy, in which males provide all parental care after fertilization in highly specialized brooding structures located on their ventral surface [10]. Recent research on the pot-bellied seahorse $(H$. abdominalis) found evidence of a single MH II $\beta$ locus with high allelic diversity in this species [11], and a highly skewed distribution of nonsynonymous variation across the gene [12]. Behavioral studies of $H$. abdominalis indicate that females can discriminate male $\mathrm{MH}$ II $\beta$ genotype using olfactory cues, and use this information when making mate choice decisions [13]. Males, in contrast, show a lack of MH II $\beta$ olfactory discrimination.

Transcriptome profiling of the seahorse indicates that this species carries a minimal MH class II system, with single expressed copies of both $\mathrm{MH} \mathrm{II} \alpha$ and $\beta$. Intriguingly, while these genes are intact in the seahorse, pipefish (Syngnathus typhle) have apparently lost $\mathrm{MH}$ class II genes as well as $\mathrm{CD}^{+}$, a critical component of MH II-associated T-helper cells [14]. Given the close relationship between seahorses and pipefish $[15,16]$, the loss of MH II function in the pipefish appears to be a relatively recent evolutionary event, one which would be expected to have an important impact on adaptive immune function in this group.

In this study, we identify the genomic structure and pattern of inheritance of major histocompatibility class II genes in the seahorse, and use population-level screening to examine the distribution of genetic variation across their peptide-binding regions. We characterize tissuespecific patterns of expression of the two loci, with particular emphasis on the male brood pouch, the reproductive organ associated with male pregnancy in this species. Finally, MH II $\alpha$ and II $\beta$ data are used to reconstruct the functionally important peptide-binding cleft of the molecule via homology modelling to the known crystallographic structure of the mature protein. Despite the tight physical linkage between MH II $\alpha$ and II $\beta$ in the seahorse, composite allele profiles reveal high levels of functional diversity at both loci, and a strong signature of intraand interlocus recombination, insights essential for understanding the structure, function and evolution of the biologically-active protein complex.

\section{Results}

\section{The seahorse possesses a minimal $\mathrm{MH}$ II system}

Full length cDNA and gDNA amplification of MH II $\alpha$ in the seahorse revealed the existence of a single four exon MH II $\alpha$ locus in this species. PCR reactions designed to bridge the full $\mathrm{MH}$ class II gene region produced two PCR products in an individual heterozygous for MH II $\beta$, both of which were successfully cloned and sequenced using primers distributed across the length of the sequence (Table 1). Analysis of cloned products identified extended single bp and tandem repeats in intronic regions of both $\mathrm{MH} \mathrm{II} \alpha$ and $\mathrm{II} \beta$, along with a large $786 \mathrm{bp}$ indel located in the intergenic region (Figure 1). The full length $\mathrm{MH}$ class II region of the seahorse is 9,130 - 9,884 bp in length, and contains a single class II $\alpha$ and II $\beta$ locus (Figure 1). Exonic variation is concentrated in the putative peptide binding regions (PBR) of the two MH loci (Figure 1), and while overall variation in coding regions is low, all exonic SNPs in the sequenced individual are non-synonymous (MH II $\alpha$ Exon 2 (PBR): 11/11 SNPs non-synonymous, MH II $\alpha$ Exon 4: 3/3, MH II $\beta$ Exon 2 (PBR): 4/4, MH II $\beta$ Exon 3: 1/1; MH II $\beta$ Exon 5: 1/1).

$\mathrm{MH}$ II $\alpha$ and II $\beta$ are constitutively expressed, and present in the brood pouch of both pregnant and nonpregnant male seahorses (Figure 2). RT-PCR analysis showed no differences in expression levels of either gene across different tissues or different stages of pregnancy.

Analysis of the MH class II $\alpha$ and II $\beta$ PBR in five families of known parentage demonstrates that genetic variation at both loci is inherited in a Mendelian fashion (Figure 3). 
Table 1 Primers $\left(5^{\prime} \rightarrow 3^{\prime}\right)$ used to amplify and sequence major histocompatibility class II genes in $\mathrm{H}_{\text {. abdominalis }}$

\begin{tabular}{|c|c|c|c|}
\hline \# & Name & Sequence $5^{\prime}-3^{\prime}$ & Location \\
\hline 1 & MHIla-E1F3 & TTACTCCGTTGCGGCGACGCC & Exon 1 \\
\hline 2 & MHIla-I1R & CGTCTGTACAATACTTCCGTAC & Intron 1 \\
\hline 3 & MHIIa-I1F & CAGTTACCAGGACAATGAC & Intron 1 \\
\hline 4 & MHIla-I1F2 & CAAAAAGCGGTGCTTATCGAG & Intron 1 \\
\hline 5 & MHIla-I1R2 & GCTTTAACTTGAGATACAAGTACC & Intron 1 \\
\hline 6 & MHIla-I1R3 & CACCAACAGTGAAAAACCACAAG & Intron 1 \\
\hline 7 & MHIla-I1F3 & CTTGCGGGGGAAACGGCGAAG & Intron 1 \\
\hline 8 & MHIla-E2F* & GACGTCATCCACACAGACATGC & Exon 2 \\
\hline 9 & MHIla-E2R2 & GATTCTAGTGGCTGATCCTTCAATGC & Exon 2 \\
\hline 10 & MHIla-E3R* & TGATGGGATGGGAGGGAGGATC & Exon 3 \\
\hline 11 & MHIla-E3F & AGACCTTCCAACTGGACTTCAC & Exon 3 \\
\hline 12 & MHIla-E3R2 & GTGAAGTCCAGTTGGAAGGTCTGG & Exon 3 \\
\hline 13 & MHIIa-I3R & CACGTTATTTATCATCGGCATAG & Intron 3 \\
\hline 14 & MHIla-E4F & CATCAAAGGAAACCAATGCAACTG & Exon 4 \\
\hline 15 & MHIIa-3UTRR & GTGAGGACATAATGTGCGCCACC & 3' UTR \\
\hline 16 & MHIla-3UTRF & GGTGGCGCACATTATGTCCTCAC & 3' UTR \\
\hline 17 & MHIIß-5UTRR5 & GCAATACACTGGGGTTCTCTG & 5' UTR \\
\hline 18 & MHIIß-5UTRF2 & CAGTGTATTGCAGAACATGG & $5^{\prime} \cup T R$ \\
\hline 19 & MHIIß-5UTRR6 & TATCGGTACTGGCCCATCC & $5^{\prime}$ UTR \\
\hline 20 & MHIIB-5UTRF & GCGACCAAAATCCGTTCCAG & 5' UTR \\
\hline 21 & MHIIß-5UTRR4 & GGCGTGTTTGTTGTGATTTACAC & $5^{\prime}$ UTR \\
\hline 22 & MHIIß-5UTRF3 & GCTTTGTGCAACTAATTGTGC & $5^{\prime} \cup T R$ \\
\hline 23 & MHII $\beta-5 U T R R 2$ & GGATCCAGTCTAATCTGAATCCCG & $5^{\prime} \cup T R$ \\
\hline 24 & MHII-5UTRR & CCAACCTGAGCACAAACTTG & $5^{\prime}$ UTR \\
\hline 25 & MHIIß-UTR5F & CAAGTTTGTGCTCAGGTTGG & 5' UTR \\
\hline 26 & $M H \| \beta-E 1 R$ & AAGGTGAGGAAAAGGAGGC & Exon 1 \\
\hline 27 & $M H \| \beta-E 1 F 2^{*}$ & GCCTCCTITTCCTCACCTTC & Exon 1 \\
\hline 28 & $M H \| \beta-E 2 R 2$ & GATGTCATTCTGGTCACTCGAGTT & Exon 2 \\
\hline 29 & $M H \| \beta-E 2 R^{*}$ & GAGCGCACTTTCGTAGTCAA & Exon 2 \\
\hline
\end{tabular}

*RT-PCR primers.
Analyses of allelic phase in parents and offspring support the tight physical linkage of MH II $\alpha$ and $\beta$, with no evidence of germline recombination evident in the dataset (Figure 3).

\section{Evidence of balancing selection on the MH Ila PBR}

Direct sequencing of the putative MH II $\alpha$ peptidebinding region in a population sample of 101 individuals identified a high level of functionally important genetic variation, with 18 alleles observed in the 246 bp region, including 25 substitutions at 24 polymorphic nucleotide sites. Nucleotide diversity $(\pi)$ in the population sample equaled $0.030 \pm 0.007$, similar to the level of genetic variation found at MH II $\beta$ of the seahorse $(\pi=0.031 \pm 0.007)$ [11]. 84\% of individuals were heterozygous for MH II $\alpha$ (85 of 101), while $16 \%$ were homozygous, values consistent with Hardy-Weinberg expectations (HWE Exact Test: $\mathrm{p}=0.61$.

All 25 observed nucleotide substitutions in the $\mathrm{MH}$ II $\alpha$ PBR were found to be non-synonymous, translating into amino acid substitutions at 22 of the 82 sites of the translated protein sequence, striking evidence of positive selection $(\mathrm{dN} / \mathrm{d} S: \infty ; \mathrm{dN}=0.039 ; \mathrm{dS}=0.000$; Z-test for positive selection: $\mathrm{p}<0.001$; Table 2 ). A site-specific analysis of nucleotide variation including positive selection (M8) fit the data significantly better than a neutral model of molecular evolution $(\mathrm{M} 7)(\mathrm{LRT}=51.507, \mathrm{df}=2, \mathrm{p}<0.001)$ and revealed significant evidence of positive selection at all 22 variable amino acid sites (Bayes Empirical Bayes Analysis; p > 99\%; Figure 4), a pattern consistent with that observed at the physically linked MH II $\beta$ locus, where coding variation is also concentrated at peptide-binding sites [11]. Inferences based on a comparison of the M1a (Nearly Neutral) and M2a (Positive Selection) models in Codeml were identical (data not shown).

\section{Network comparison reveals the existence of composite} recombinants

An allelic network was constructed to visualize relationships among the $18 \mathrm{MH}$ II $\alpha$ alleles and their relative frequencies (Figure 5a). The structure of the MH II $\alpha$

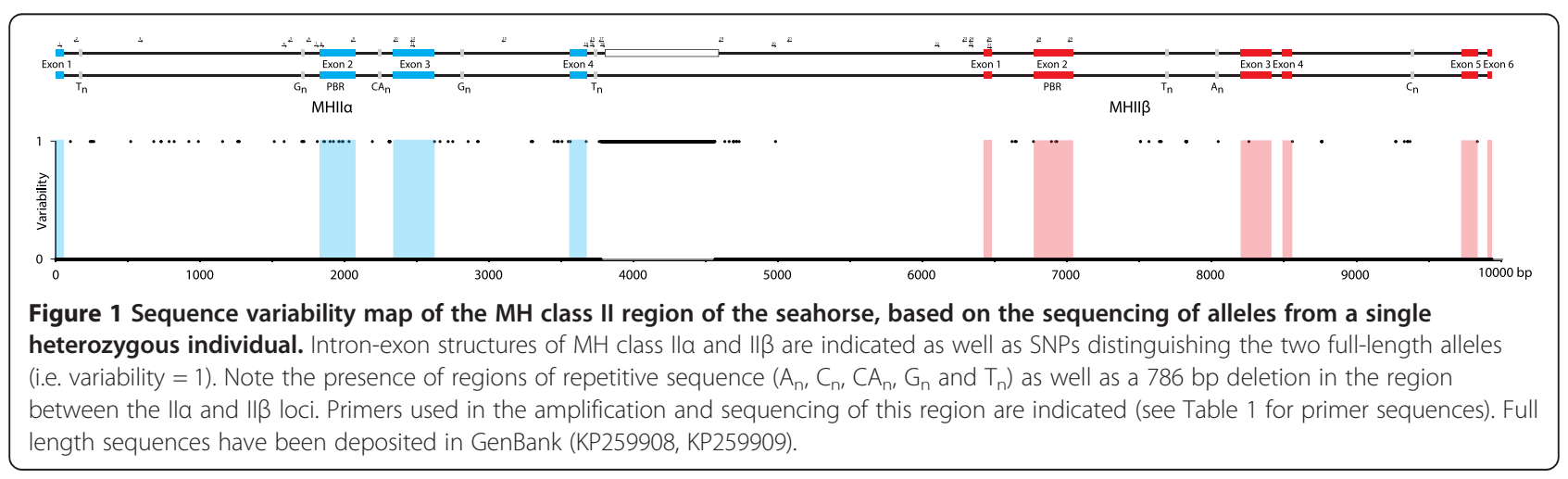




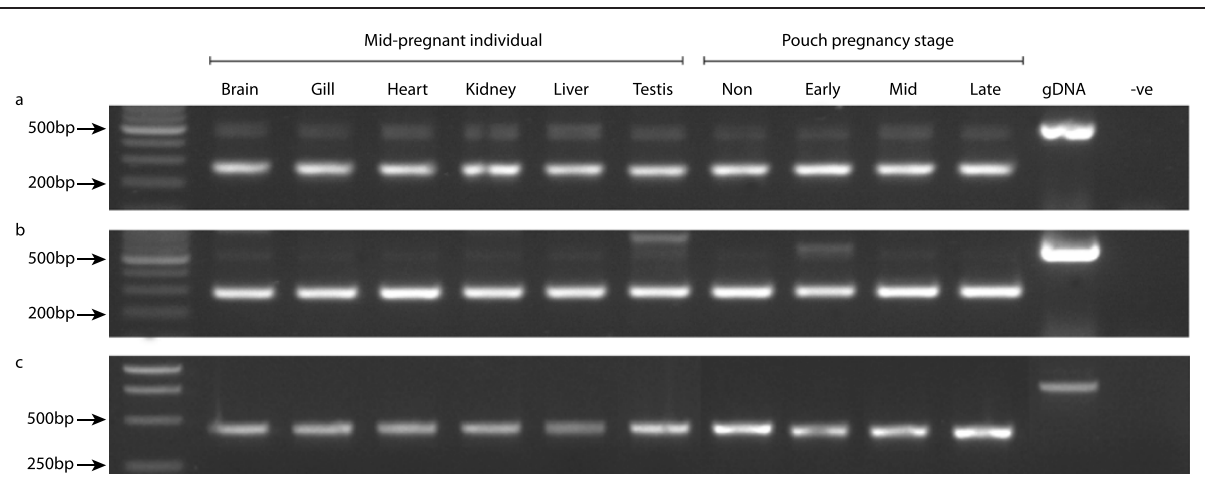

Figure $2 \mathrm{MH}$ class Ila and $I \mathrm{\beta}$ genes are ubiquitously expressed in the seahorse. Gel images showing results of RT-PCR on a range of seahorse tissues: (a) MH class Ila, (b) MH class II, (c) Beta-actin (positive control).

network is similar to that observed at MH II $\beta$, with a small number of common alleles (DAA-E2*02, 03, 05 and 10), and a larger number of low frequency alleles present in $\leq 10$ copies in the population sample (Figure 5).

An analysis of genetic linkage between MH II $\alpha$ and II $\beta$ alleles in the population sample supported the results of the parentage analysis, and indicated significant linkage disequilibrium between the two loci $\left(x^{2}=469.70\right.$; $\mathrm{p}<0.001)$. With 18 alleles detected at MH II $\alpha$ and 17 alleles at MH II $\beta$, a minimum of 18 composite alleles and maximum of $18 * 17=306$ alleles could be realized in the population sample. The observation of 36 composite $\mathrm{MH}$ II $\alpha / \mathrm{II} \beta$ alleles in the population dataset (Figure 5c) reflects a significant history of recombination in this region.

\section{Evidence of intra- and interlocus recombination}

A statistical analysis of recombination at MH II $\alpha$ failed to detect significant evidence of intralocus recombination $(p=0.077)$. An analysis of the phased MH II $\alpha / I I \beta$ dataset, in contrast, found a strong signature of recombination $(\mathrm{p}=0.0002)$, with $8 \mathrm{MH} \mathrm{II} \alpha / \mathrm{II} \beta$ recombinants and 2 of the $3 \mathrm{MH}$ II $\beta$ intralocus recombinant genotypes

\begin{tabular}{|c|c|c|c|c|c|c|c|c|c|c|}
\hline \multirow{2}{*}{ Individual } & \multicolumn{2}{|c|}{ Family A } & \multicolumn{2}{|c|}{ Family B } & \multicolumn{2}{|c|}{ Family C } & \multicolumn{2}{|c|}{ Family D } & \multicolumn{2}{|c|}{ Family E } \\
\hline & MH Il $\alpha$ & MH $॥ \beta$ & MH II $\alpha$ & MH $\| \beta$ & MH II $\alpha$ & MH $\| \beta$ & MH II $\alpha$ & MH ॥ $\beta$ & MH II $\alpha$ & MH $\| \beta$ \\
\hline \multirow{2}{*}{ Father } & 2 & 4 & 2 & 6 & 3 & 13 & 2 & 4 & 3 & 2 \\
\hline & 3 & 3 & 3 & 2 & 8 & 5 & 2 & 9 & 2 & 6 \\
\hline \multirow{2}{*}{ Mother } & 2 & 4 & 3 & 1 & 2 & 8 & 2 & 6 & 3 & 1 \\
\hline & 2 & 15 & 2 & 8 & 3 & 2 & 7 & 16 & 6 & 11 \\
\hline \multirow{2}{*}{ Juvenile 1} & 2 & 4 & 2 & 6 & 3 & 13 & 2 & 4 & 2 & 6 \\
\hline & 2 & 4 & 3 & 1 & 3 & 2 & 2 & 6 & 6 & 11 \\
\hline \multirow{2}{*}{ Juvenile 2} & 3 & 3 & 2 & 6 & 8 & 5 & 2 & 4 & 3 & 2 \\
\hline & 2 & 15 & 2 & 8 & 3 & 2 & 7 & 16 & 6 & 11 \\
\hline \multirow{2}{*}{ Juvenile 3} & 2 & 4 & 2 & 6 & 8 & 5 & 2 & 9 & 2 & 6 \\
\hline & 2 & 15 & 3 & 1 & 3 & 2 & 7 & 16 & 3 & 1 \\
\hline \multirow{2}{*}{ Juvenile 4} & 2 & 4 & 2 & 6 & 3 & 13 & 2 & 4 & 3 & 2 \\
\hline & 2 & 15 & 3 & 1 & 2 & 8 & 2 & 6 & 6 & 11 \\
\hline \multirow{2}{*}{ Juvenile 5} & 3 & 3 & 2 & 6 & 8 & 5 & 2 & 9 & 2 & 6 \\
\hline & 2 & 15 & 2 & 8 & 2 & 8 & 7 & 16 & 3 & 1 \\
\hline \multirow{2}{*}{ Juvenile 6} & 3 & 3 & 3 & 2 & 3 & 13 & 2 & 9 & 3 & 2 \\
\hline & 2 & 15 & 3 & 1 & 2 & 8 & 2 & 6 & 3 & 1 \\
\hline \multirow{2}{*}{ Juvenile 7} & 3 & 3 & 2 & 6 & 3 & 13 & 2 & 9 & 3 & 2 \\
\hline & 2 & 4 & 2 & 8 & 2 & 8 & 2 & 6 & 3 & 1 \\
\hline \multirow{2}{*}{ Juvenile 8} & 2 & 4 & 2 & 6 & 3 & 13 & 2 & 4 & 3 & 2 \\
\hline & 2 & 4 & 2 & 8 & 2 & 8 & 7 & 16 & 6 & 11 \\
\hline \multirow{2}{*}{ Juvenile 9} & & 3 & 3 & 2 & 8 & 5 & & & & \\
\hline & 2 & 15 & 3 & 1 & 2 & 8 & & & & \\
\hline \multirow{2}{*}{ Juvenile 10} & 3 & 3 & & & & & & & & \\
\hline & 2 & 15 & & & & & & & & \\
\hline \multirow{2}{*}{ Juvenile 11} & 3 & 3 & & & & & & & & \\
\hline & 2 & 4 & & & & & & & & \\
\hline \multirow[t]{2}{*}{ Juvenile 12} & 3 & 3 & & & & & & & & \\
\hline & 2 & 15 & & & & & & & & \\
\hline \multirow{2}{*}{ Juvenile 13} & 2 & 4 & & & & & & & & \\
\hline & 2 & 15 & & & & & & & & \\
\hline
\end{tabular}

Figure 3 Mendelian inheritance of $\mathbf{M H}$ class II alleles in five families of known parentage. Parental alleles are color-coded for ease of discrimination in offspring (Paternal alleles: light/dark blue; Maternal alleles: light/dark red). Blockwise inheritance of parental MH alleles provides direct evidence of tight physical linkage. 
Table 2 Synonymous and non-synonymous substitution rates for exon 2 alleles of the seahorse MH class Ila gene

\begin{tabular}{lllllll}
\hline Locus & Length $(\mathbf{b p})$ & Samples & Alleles & dN & dS & dN/dS \\
\hline Exon 2 & 246 & 101 & 18 & 0.039 & 0.000 & $\infty^{* *}$ \\
Exon 2, PBS & 60 & 101 & 17 & 0.110 & 0.000 & $\infty^{*}$ \\
Exon 2, non-PBS & 186 & 101 & 11 & 0.018 & 0.000 & $\infty^{*}$ \\
\hline
\end{tabular}

Probabilities $\left({ }^{*}<0.05,{ }^{* *}<0.001, \mathrm{~ns}=\right.$ not significant) are derived from a Z-test (H1 = positive selection). Peptide-binding sites (PBS) refer to the human sites, identified by crystallographic analysis [3] (Figure 4).

identified in an earlier analysis of MH II $\beta$ PBR variation [11] (Table 3).

\section{Protein structure}

The inferred quaternary structure of the seahorse $\mathrm{MH}$ class II molecule closely resembles that of the mouse model, with a clearly defined peptide binding groove formed by the interaction of MH class II $\alpha$ and II $\beta$ loci (Figure 6). While the majority of positively selected sites in the seahorse are located in this peptide-binding groove, several selected sites at both the II $\alpha$ (LYS.39 $\alpha$ and GLN.40 $\alpha$ ) and II $\beta$ loci (LEU.18 $\beta$ and ASN.61 $\beta$ ) are located away from this groove on the surface of the mature peptide.

\section{Discussion}

Adaptive immune diversity at the minimal $\mathrm{MH}$ class II system of the seahorse is enhanced by high levels of genetic variation at both MH II $\alpha$ and II $\beta$, highlighting the importance of considering both loci in order to understand
MH class II function in this system. Intra- and interlocus recombination act to further augment genotypic variation, generating novel genotypes despite the tight physical linkage of MH class II loci in the seahorse.

Despite high levels of functionally important genetic diversity and clear evidence of positive selection in the PBR of MH II $\alpha$, this region shows a complete absence of synonymous substitutions. The significant excess of nonsynonymous substitutions relative to synonymous variation is also characteristic of the PBR of MH II $\beta$ [11], and low levels of genetic variation are found in the other coding regions of the two genes [Figure 1;12]. While the high number of non-synonymous variants in the PBR is expected given its role in antigen recognition, the low levels of synonymous substitutions suggest that selective sweeps driven by positively selected sites may act to homogenize neutral variation through a process of genetic hitchhiking [12]. New mutations at sites involved in antigen detection that provide a selective advantage in host-pathogen interactions are expected to rapidly increase in frequency [17],

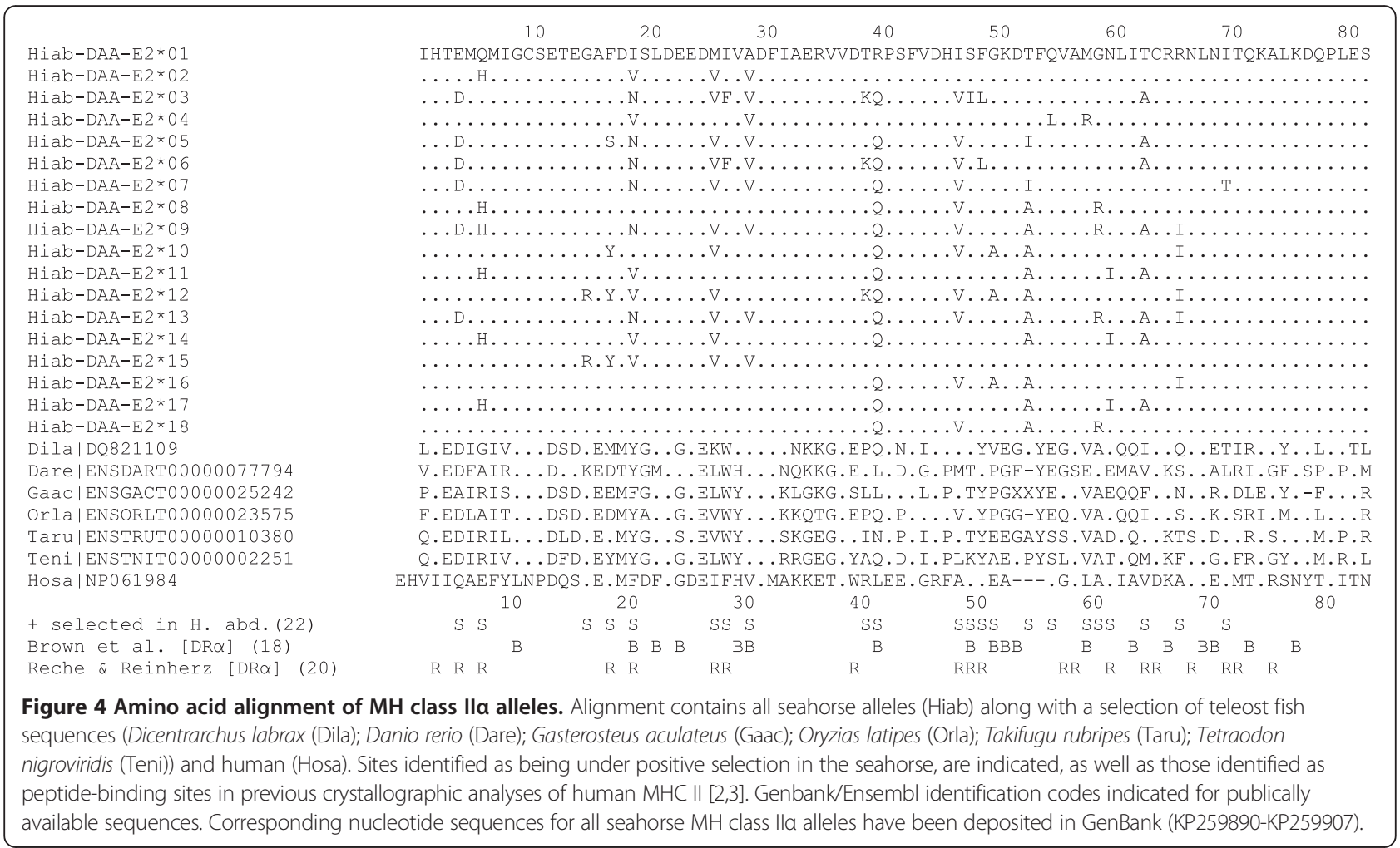


a

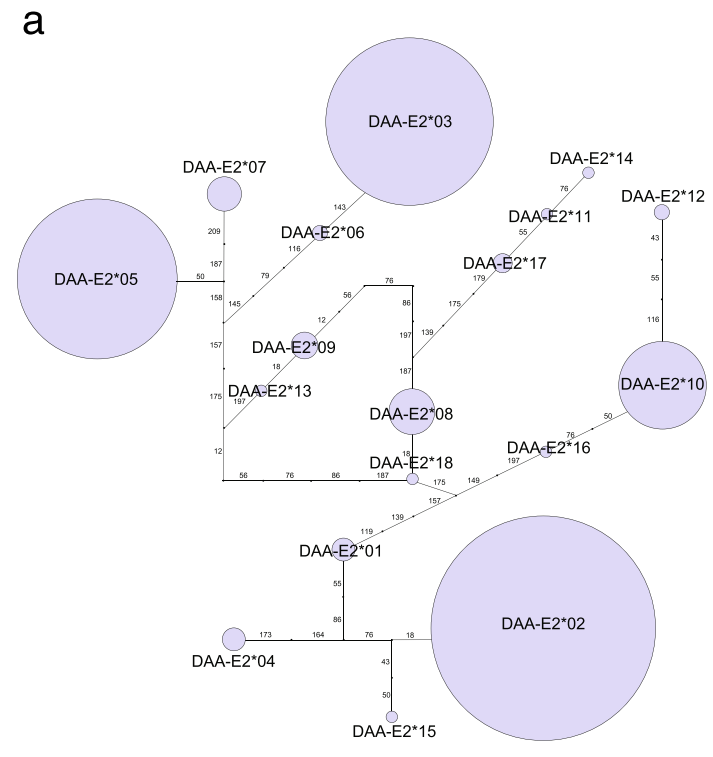

C

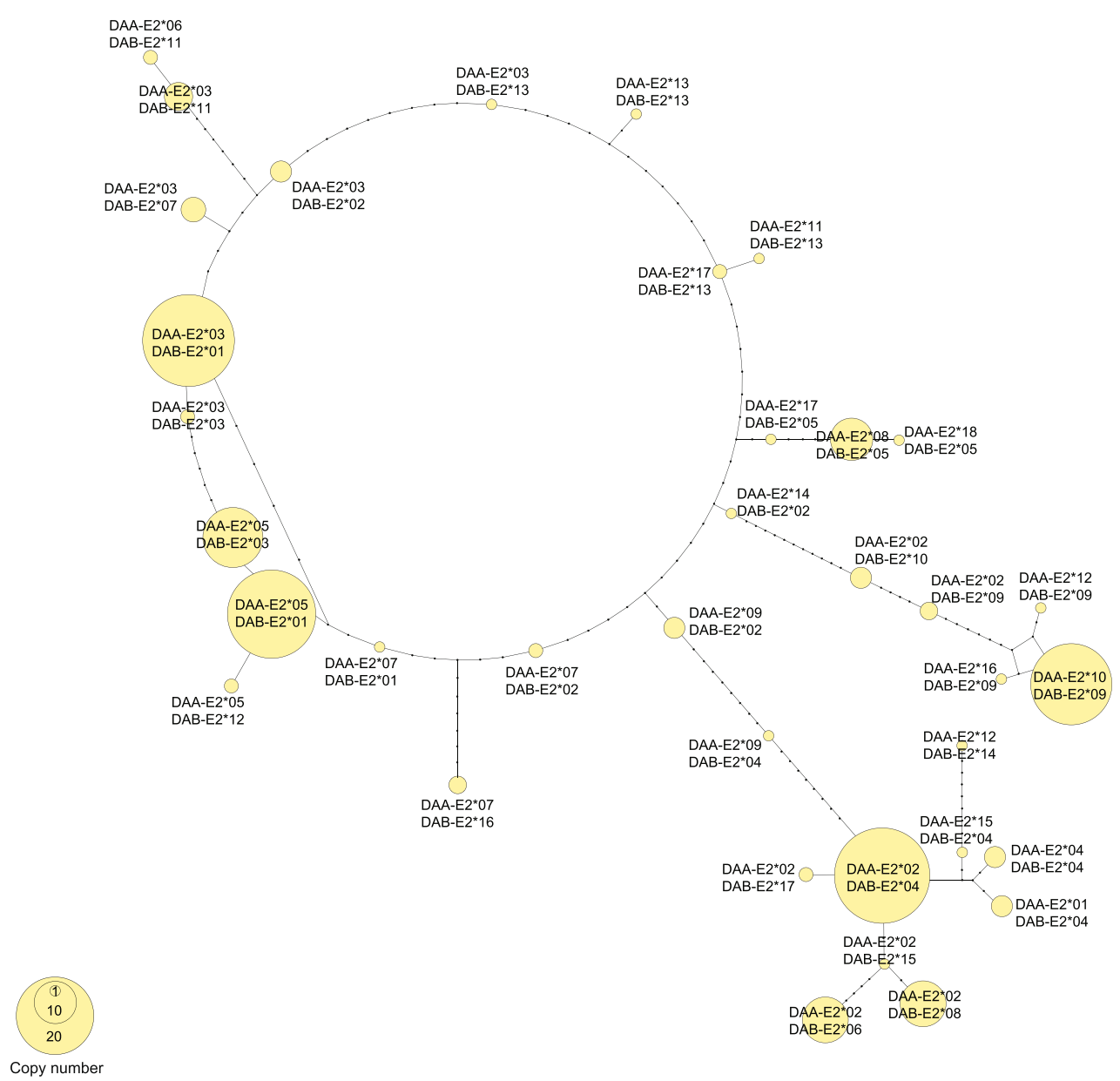

Figure 5 Allelic networks of $\mathrm{MH}$ class II variation ( $\mathrm{n}=101$ individuals). Figure shows allelic variability in the pattern of non-synonymous substitutions across exon 2 of (a) $\mathrm{MH}$ class $\|$ la and (b) $\mathrm{MH}$ class $\| \beta$, the immunologically-active region of the two genes; (c) Composite $\mathrm{MH}$ network constructed from phased genotype data. Note the presence of reticulation in the MH $\| \beta$ network, evidence of intralocus recombination [11]. Reticulation is also evident in the composite allele network. 
simultaneously increasing the frequency of linked neutral variants. Subsequent short-tract gene conversion and recombination may enhance functional diversity at $\mathrm{MH}$ peptide binding sites, without increasing neutral genetic variation. Over time, the hitchhiking of linked genetic variants with positively selected sites may erode local neutral variation, and impact functionally important genetic variation at neighboring loci. The combination of positive selection and genetic hitchhiking is likely responsible for the highly skewed $\mathrm{dN} / \mathrm{dS}$ ratios observed here.

Amino acids experiencing positive selection at both MH II $\alpha$ and II $\beta$ are preferentially located at sites predicted to be directly involved in antigen binding (Figure 4), as inferred from homology modelling to the experimentally determined crystallographic structures of human and mouse MHC $[3,18]$. The presence of several positively selected sites outside this region (Figure 6) suggests potential differences in the quaternary structure of the mature protein in vivo and/or additional selective constraints acting away from the main peptide binding groove of the seahorse.

Despite the tight physical linkage of MH class II loci in the seahorse, both intra- and interlocus recombinants were detected in the population sample, a finding which likely reflects the selective advantages of novel allelic variants generated by recombination. While empirical recombination rates are not available for the seahorse, a rough approximation based on the average recombination rate in the human genome (ca. $1 \mathrm{cM} / \mathrm{Mb}$ ) would suggest that a crossover event in the 5,000 bp interval separating the PBRs of MH II $\alpha$ and II $\beta$ would be expected every 20,000 meioses. Recombination is likely to be even more common in the $\mathrm{MH}$ region, as hotspots have been identified in the MH class II regions of humans [19] and mice [20] that have been shown to provide an

Table 3 Inferred recombinant genotypes from analysis of the phased MH class IIa/II $\beta$ PBR dataset

\begin{tabular}{llll}
\hline Genotype & $\begin{array}{l}\text { Recombination } \\
\text { breakpoint }\end{array}$ & $\begin{array}{l}\text { Mutation } \\
\text { savings }\end{array}$ & p-val \\
\hline DAA-E2*9/DAB-E2*4 & $198-4896$ & 9 & 0.0001 \\
DAA-E2*3/DAB-E2*13 & $180-4896$ & 9 & 0.0002 \\
DAA-E2*12/DAB-E2*14 & $188-4890$ & 7 & 0.0002 \\
DAA-E2*13/DAB-E2*13 & $198-4890$ & 6 & 0.0001 \\
DAA-E2*7/DAB-E2*2 & $210-5007$ & 6 & 0.0016 \\
DAA-E2*2/DAB-E2*6 & $5058-5079$ & 6 & 0.0004 \\
DAA-E2*9/DAB-E2*2 & $198-4896$ & 6 & 0.0001 \\
DAA-E2*2/DAB-E2*9 & $193-5007$ & 5 & 0.0021 \\
DAA-E2*2/DAB-E2*10 & $4930-5007$ & 5 & 0.0001 \\
DAA-E2*14/DAB-E2*2 & $180-4896$ & 5 & 0.0053 \\
\hline
\end{tabular}

Note: Recombination breakpoints for interlocus recombinants include $4630 \mathrm{bp}$ separating the sequenced peptide binding regions of MHIla and $I \beta$ (see Figure 1). important source of allelic variation at these loci. Sperm profiling indicates that gene conversion, the unidirectional exchange of short sequence tracts without crossover, may make up as much as $90 \%$ of all recombination events at MH class II loci [21], and serve a particularly important role in generating new PBR variants from standing genetic variation. Once new variants are produced, they may rapidly spread through the population if they offer selective advantages relative to existing allelic variation [17].

While analyses of linkage disequilibrium in population samples provide evidence of breakpoints of historical recombination events, the estimation of recombination rates requires the identification of germline recombinants from pedigree data and/or the direct typing of gametes. No MH II $\alpha / \mathrm{II} \beta$ recombinants were identified in the parentage analysis carried out as part of this study, but the modest size of this dataset had limited power to identify meiotic recombination. Although seahorses have reduced testes and produce low amounts of sperm [22], sperm typing could provide a high throughput method to estimate meiotic recombination rates, and to explore the relative importance of gene conversion and classical recombination in structuring patterns of genetic variation across the major histocompatibility region of the seahorse. An understanding of baseline recombination rates would also facilitate more detailed analyses of the potential selective advantages of novel recombinant genotypes.

Studies of MH class II genetic variability in non-model organisms have been heavily biased towards MH II $\beta$, an approach which has been informed by the low variability of $\mathrm{MH}$ II $\alpha$ relative to $\mathrm{MH}$ II $\beta$ in humans. Large scale population genotyping of the 5 classical $\mathrm{MH}$ II $\alpha$ and II $\beta$ genes in more than 20,000 individuals has identified $>2,750 \mathrm{MH}$ II $\beta$ alleles and only $116 \mathrm{MH}$ II $\alpha$ variants, a greater than $20 \times$ excess of $\mathrm{MH}$ II $\beta$ allelic diversity relative to MH II $[6]$.

Gomez [9] carried out the first integrative analysis of MH II $\alpha$ and II $\beta$ variation in a non-model species, simultaneously characterizing genetic variation at both loci of the minimal MH system of salmonid fishes. An analysis of genetic variation in population samples of three salmonid species revealed similar levels of variation at both loci $(\mathrm{MH} \mathrm{II} \alpha / \mathrm{II} \beta$ alleles - Oncorhynchus mykiss $(\mathrm{n}=40)$ : 10/11; Oncorhynchus kisutch $(\mathrm{n}=75): 4 / 8$; Salmo salar ( $\mathrm{n}=27): 9 / 9)$, a result which led the authors to suggest that high genetic variability at MH II $\alpha$ could allow species with a minimal $\mathrm{MH}$ system to mount a successful immune response without the additional genetic variation associated with multiple gene copies [9].

Recent work suggests that the high genetic variability of MH II $\alpha$ may not be restricted to species with a minimal MH system such as salmonids or seahorses, 

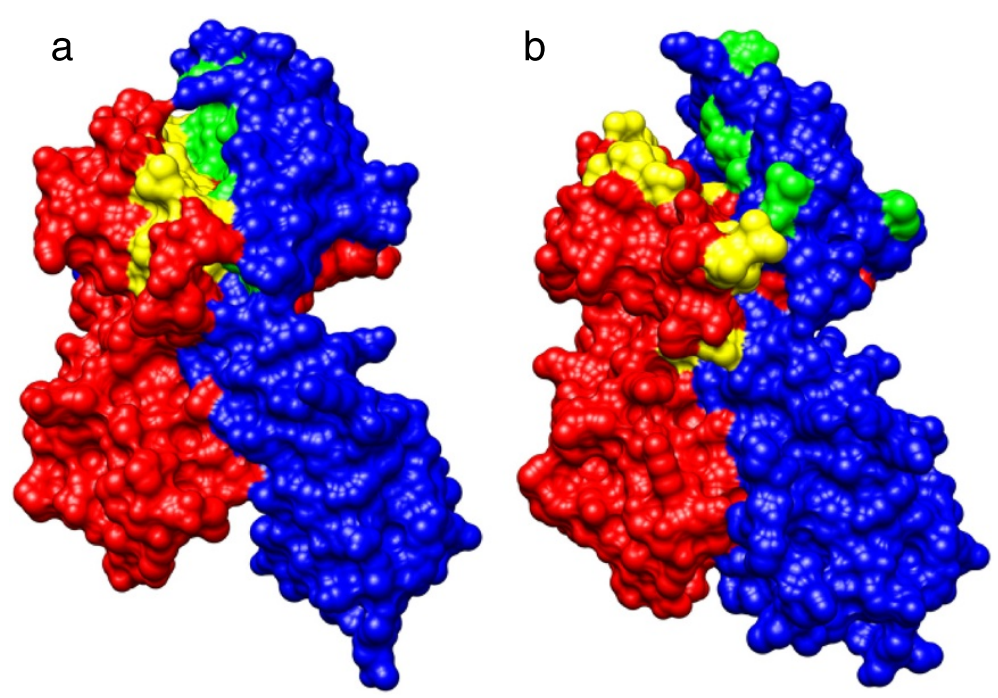

Figure 6 Positively selected sites of the seahorse MH class II are concentrated in the inferred peptide binding groove of the mature protein. Quaternary structure models of the major histocompatibility class II molecule for (a) Mus musculus and (b) Hippocampus abdominalis, based on crystallographic reconstructions for Mus (pdb1es0). Surface structures of extracellular components of MH Ila (red) and MH II (blue) are shown, as are (a) peptide-binding sites, and (b) sites inferred to be under positive selection in the seahorse (yellow and green for $\mathrm{MH}$ lla and $\| \beta$ respectively).

but may be evolutionarily widespread. A recent population survey ( $\mathrm{n}=367$ wild-caught individuals) of single copies of $\mathrm{MH} \mathrm{II} \alpha(\mathrm{H}-2 \mathrm{~A} a)$ and $\mathrm{II} \beta(\mathrm{H}-2-\mathrm{E} b)$ in the two locus system of the house mouse (Mus musculus) recovered $27 \mathrm{MH}$ II $\alpha$ and $17 \mathrm{MH}$ II $\beta$ alleles, an excess of $\mathrm{MH}$ II $\alpha$ allelic variability relative to $\mathrm{MH}$ II $\beta$ [23]. Similarly, a large genotyping study of two $\mathrm{MH}$ loci in Japanese domestic cattle $(\mathrm{n}=650)$ detected 15 and 26 alleles at MH II $\alpha$ (DQA1) and $\mathrm{MH}$ II $\beta$ (DRB3) loci, respectively [24]. Taken together, the results of these recent studies suggest that high genetic diversity at MH II $\alpha$ is likely to be widespread, and is not a phenomenon restricted to species with a minimal MH class II system. As such, studies which continue to focus exclusively on variation at MH II $\beta$ may be missing an important component of ecologically relevant variation, providing an incomplete picture of how selective pressures influence genetic diversity of the biologically active protein dimer.

Unfortunately, while it is relatively straightforward to characterize $\mathrm{MH}$ diversity using traditional Sanger sequencing in species carrying a minimal $\mathrm{MH}$ system, researchers working on more complex $\mathrm{MH}$ systems have been unable to accurately screen $\mathrm{MH}$ allelic diversity and classify variation to specific loci, an essential prerequisite for more detailed investigations of selection. Recent innovations in next-generation sequencing [25], coupled with rapidly maturing statistical approaches for processing population data for multigene families such as the MHC $[26,27]$, offer an exciting new avenue for high-throughput systems-based analyses of immunological variation. While such studies are still in their infancy, they show great promise for population-level analyses of $\mathrm{MH}$ loci e.g. [28-30]. Thus far, studies have followed the design of earlier research in focusing on $\mathrm{MH} \mathrm{II} \beta$, but combined analyses of MH II $\alpha$ and II $\beta$ should be straightforward once these new technologies have fully matured.

\section{Conclusions}

Antigen recognition by the adaptive immune system of the seahorse is enhanced by high levels of variability at both $\mathrm{MH}$ class II $\alpha$ and II $\beta$ loci. Strong positive selection on sites involved in pathogen recognition, coupled with high levels of intra- and interlocus recombination, generate a patchwork distribution of genetic variation essential for understanding the functional diversity of the mature protein.

While studies of MH diversity in non-model organisms are now widespread, the majority of investigations have focused exclusively on $\mathrm{MH}$ class $\mathrm{II} \beta$, an approach that has been justified by the relatively low levels of variation observed at $\mathrm{MH}$ class II $\alpha$ loci in humans. Our results suggest that humans may be atypical in this regard, a conclusion supported by recent comparative analyses of MH class II diversity in both mammals and fish, which indicate that genetic diversity at $\mathrm{MH}$ class II $\alpha$ loci may often meet or exceed that observed at class II $\beta$. Given the high rates of intra- and interlocus recombination found at $\mathrm{MH}$ loci, studies aiming to link patterns of MH class II diversity to survival and reproduction should consider the structure and function of the immunologically active protein molecule. In failing to do so, 
researchers may be unintentionally overlooking an important component of ecologically relevant genetic variation.

\section{Methods \\ Sample collection}

Adult seahorses (H. abdominalis) were purchased from a commercial breeding facility (Seahorse Australia, Beauty Point, Tasmania) and held at the University of Zurich under an animal care and experimentation permit from the Veterinäramt Zürich (Permit 103/2008). In addition, fin clips from 5 wild-caught seahorses from Sydney, Australia ( 2 individuals collected in 2003) and Tasmania (3 individuals collected from 3 populations in 2003 and 2004) [31] were included in analyses of $\mathrm{MH}$ class II genetic diversity.

\section{Gene discovery via high-throughput transcriptome profiling}

A full plate of 454 sequencing of transcriptome libraries prepared from pouch and reference (brain, gills, liver, heart, kidney and testes) tissues from a single pregnant and non-pregnant seahorse using the GS FLX Titanium Chemistry (Roche) recovered six partial transcripts of the MH II $\alpha$ locus of the seahorse, which were assembled into a single 541 bp contig spanning the $5^{\prime}$ UTR, complete exons 1 and 2 and partial exon 3. Further details on this transcriptome screen are available elsewhere [11]. A TBLASTX search of the nucleotide collection of GenBank identified MH class II $\alpha$ of Dicentrarchus labrax as the top hit (DQ821109.1: e-value $=3 \mathrm{e}-47$ ), followed by class II $\alpha$ genes from other teleost species (Morone saxatilis L35062.1: e-value $=8 \mathrm{e}-42$, Larimichthys crocea EF681861.1: e-value = 5e-41, Miichthys miiuy GU936787.1: e-value $=2 \mathrm{e}-40$, and Epinephelus coioides GU992883.1: $\mathrm{e}$-value $=3 \mathrm{e}-40)$.

\section{Full-length cDNA sequencing}

Total RNA was extracted from brain, gill, kidney, liver, pouch and testes tissues of two pregnant seahorses with RNeasy extraction columns (Qiagen). RNA extractions were subsequently DNase treated, standardized to a common concentration of $85 \mathrm{ng}$, and pooled for library construction. $5^{\prime}$ and $3^{\prime}$ RACE libraries were prepared from $1 \mu \mathrm{g}$ of total RNA using a SMARTer RACE cDNA amplification kit (Clontech).

The full-length cDNA sequence of the MH class II $\alpha$ gene of the seahorse was obtained using separate $5^{\prime}$ and 3' RACE reactions primed with gene-specific primers

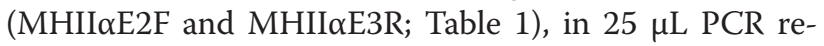
actions using $1.5 \mu \mathrm{L}$ of $5^{\prime} / 3^{\prime}$ RACE-ready cDNA. Both reactions produced single products from the $5^{\prime}$ and $3^{\prime}$ ends of the class II $\alpha$ gene, which were PCR-purified with Montage $\mathrm{PCR} \mu_{96}$ Filter Plates (Millipore) and eluted in

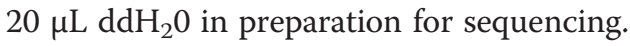

Sequencing reactions were carried out in $10 \mu \mathrm{L}$ volumes consisting of $2-4 \mu \mathrm{L}$ purified PCR product, $0.2 \mu \mathrm{M}$ primer, and $1 \mu \mathrm{L}$ Big Dye v3.1 Terminator Cycle Sequencing mixture (Applied Biosystems). Cycling conditions consisted of 30 cycles of $10 \mathrm{sec}$ at $96^{\circ} \mathrm{C}, 5 \mathrm{sec}$ at $50^{\circ} \mathrm{C}$ and $4 \mathrm{~min}$ at $60^{\circ} \mathrm{C}$. Ethanol-purified products were sequenced on an ABI 3730 automated sequencer (Applied Biosystems).

\section{Full-length genomic sequencing of the Major Histocompatibility class II gene region}

To elucidate the structure and distribution of variation across the MH region of the seahorse, genomic DNA of a single non-pregnant individual was extracted from muscle tissue (DNeasy, Qiagen). The quality of extracted DNA was assessed on a $1.5 \%$ agarose gel and spectrophotometrically quantified using a Nanodrop 2000 (Thermo Scientific). The gDNA sequence of the $\mathrm{MH}$ class II $\beta$ for this individual has been previously published [12].

The full-length gDNA sequence of the seahorse $\mathrm{MH}$ class II gene region was determined using long-range PCR with gene-specific primers designed from the cDNA sequence of MH class II $\alpha$ and the 5' UTR region of the class II $\beta$ locus (MHII $\alpha-E 1 F 3 / M H I I \beta-5 U T R R 4 ;$ Table 1).

Long range PCR was performed in a $25 \mu \mathrm{L}$ volume containing 3U LongAmp Taq (New England Biolabs), 1× LongAmp reaction buffer, $0.4 \mathrm{mM}$ dNTPs, $0.2 \mu \mathrm{M}$ primers and 250 ng DNA. PCR amplification involved a 2 min denaturation step at $92^{\circ} \mathrm{C}$, followed by 30 cycles of $92^{\circ} \mathrm{C}(20 \mathrm{~s}), 65^{\circ} \mathrm{C}(20 \mathrm{~s})$ and $65^{\circ} \mathrm{C}(10 \mathrm{~min})$, and a final extension step for $10 \mathrm{~min}$ at $65^{\circ} \mathrm{C}$. The PCR reaction was filter-purified in preparation for cloning.

$4 \mu \mathrm{L}$ of purified PCR product was cloned into a TOPO TA cloning vector (Invitrogen) following the manufacturers' recommendations. Following overnight culture of transformed chemically competent $E$. coli at $37^{\circ} \mathrm{C}$, 5 positive colonies were picked and grown for $16 \mathrm{~h}$ in liquid culture on a $200 \mathrm{rpm}$ horizontal shaker at $37^{\circ} \mathrm{C}$. Liquid cultures were purified for downstream sequencing using a QIAprep Spin Miniprep kit (Qiagen).

Screening of plasmid DNA with primers for the hypervariable peptide binding region of the seahorse $\mathrm{MH}$ class II $\alpha$ peptide binding region revealed the presence of two alleles, both of which were sequenced to completion using a nested sequencing strategy involving primers distributed across the full length of the amplified region (Table 1) using the protocols outlined above. DNA sequencing revealed a 786 bp deletion between MH class II $\alpha$ and II $\beta$ in one of the two full-length alleles (Figure 1).

The intervening non-coding sequence between the class II $\alpha$ and II $\beta$ loci was PCR-amplified and sequenced from genomic DNA using two sets of PCR reactions, one using MHII $\alpha$-E4F/MHII $\beta$-5UTRR2 $\left(60^{\circ} \mathrm{C}\right.$ anneal $)$ and a second using MHII $\beta$-5UTRF2/MHII $\beta$-E2R2 $\left(55^{\circ} \mathrm{C}\right.$ 
anneal), both of which spanned the deletion region, allowing the determination of allelic phase of sequences from II $\alpha$ and II $\beta$ loci.

\section{RT-PCR screening of tissue-specific expression}

Samples were obtained from captive $H$. abdominalis individuals (Seahorse Australia, Beauty Point, Australia) preserved in RNAlater (Sigma-Aldrich) and then stored at $-80^{\circ} \mathrm{C}$. Four reproductively active adult males were screened for MH II gene activity, with the stage of pregnancy estimated using a recently published developmental key for syngnathid fishes [32]. Total RNA was extracted from a panel of tissues (brain, gill, heart, kidney, liver, pouch, testis from one mid-pregnant animal; and pouch from one non-pregnant, one early pregnant, and one late pregnant individual) using an RNeasy Mini Kit with QiaShredder (Qiagen) and DNase I (Invitrogen) digestion. First-strand cDNA was synthesized with SuperScript III First-Strand Synthesis System for RTPCR (Invitrogen) using random hexamer priming and 200 ng of RNA.

$\mathrm{MH}$ class II PCRs were carried out using intronspanning primers indicated in Table 1. Beta-actin (ACTB) was used as the positive control to ensure uniform amplification for each tissue, and was amplified using ACTB-E2F - GTCATGGTCGGCATGGGAC and ACTBE3R - AGGTAGTCTGTGAGGTCTCG. PCR reactions for ACTB were performed in $20 \mu \mathrm{l}$ volumes containing $0.5 \mathrm{U}$ Taq (New England Biolabs), $1 \times$ NEB reaction buffer, $0.75 \mu \mathrm{M} \mathrm{MgCl}_{2}, 0.2 \mathrm{mM}$ dNTPs, $0.2 \mu \mathrm{M}$ primers, and $1.0 \mu \mathrm{l} \mathrm{DNA}$, with the following PCR cycling conditions: $95^{\circ} \mathrm{C}$ for $1: 30$, then 30 cycles of $95^{\circ} \mathrm{C}$ for $0: 30,53^{\circ} \mathrm{C}$ for $0: 30,68^{\circ} \mathrm{C}$ for $1: 00$, followed by $68^{\circ} \mathrm{C}$ for $5: 00$. MH amplifications were performed in $25 \mu \mathrm{l}$ volumes containing $1 \mathrm{U}$ Taq (NEB), $1 \times$ NEB reaction buffer, $1.0 \mu \mathrm{M} \mathrm{MgCl}$, $0.4 \mathrm{mM}$ dNTPs, $0.2 \mu \mathrm{M}$ primers and $2.5 \mu \mathrm{l}$ DNA, with the following cycling parameters: $92^{\circ} \mathrm{C}$ for $0: 10$, then 40 cycles of $92^{\circ} \mathrm{C}$ for $0: 10,55^{\circ} \mathrm{C}$ for $0: 30,68^{\circ} \mathrm{C}$ for $2: 00$ (for $\mathrm{MH} \mathrm{II \alpha )}$ or $92^{\circ} \mathrm{C}$ for $5: 00$, then 40 cycles of $92^{\circ} \mathrm{C}$ for $0: 30,62^{\circ} \mathrm{C}$ for $0: 30,68^{\circ} \mathrm{C}$ for $4: 00$, followed by $68^{\circ} \mathrm{C}$ for 15:00 (for MH II $\beta$ ).

PCR products were subjected to electrophoresis at $100 \mathrm{~V}$ for $20 \mathrm{~min}$ in $1.5 \%$ agarose gels stained with ethidium bromide, and visualized using an AlphaImager gel documentation system (Alpha Innotech).

\section{MH Ila inheritance and MH II linkage analysis}

$\mathrm{MH}$ class II $\alpha$ Exon 2, containing the immunologically active peptide-binding region of the gene, was PCRamplified and sequenced in a sample of $47 \mathrm{~F} 1$ individuals from 5 families ( $\mathrm{n}=8-13$ per family) which had previously been characterized for patterns of genetic variation at the MHII $\beta$ peptide-binding region [11]. A comparison of parent-offspring genotype profiles allowed the inference of the mode of MHII inheritance and a means to test for linkage of II $\alpha$ and II $\beta$ loci in this species.

Standard PCR was performed in $25 \mu \mathrm{L}$ volumes containing $1 \mathrm{U}$ Taq (NEB), $1 \times$ NEB reaction buffer, $1.0 \mu \mathrm{M}$ $\mathrm{MgCl}_{2}, 0.4 \mathrm{mM}$ dNTPs, $0.2 \mu \mathrm{M}$ of either MHII $\alpha-\mathrm{E} 2 \mathrm{~F} /$ MHII $\alpha$-E3R or MHII $\alpha$-I1F3/MHII $\alpha-E 3 R$ and 25-250 ng DNA. PCR amplifications involved a $10 \mathrm{~s}$ denaturation step at $92^{\circ} \mathrm{C}$, followed by 40 cycles of $92^{\circ} \mathrm{C}(10 \mathrm{~s}), 55^{\circ} \mathrm{C}$ $(30 \mathrm{~s})$ and $68^{\circ} \mathrm{C}(2 \mathrm{~min})$. All individuals were PCRamplified and sequenced using both sets of primer pairs. PCR amplifications of both PCR purification and sequencing followed that outlined for the cDNA experiment above, producing the full length sequence of the $249 \mathrm{bp}$ exon. After trimming $2 \mathrm{bp}$ from the $5^{\prime}$ end of the sequence alignment and $1 \mathrm{bp}$ from the $3^{\prime}$ terminus to exclude incomplete amino acids, the analyzed exon 2 dataset included $246 \mathrm{bp} / 82$ amino acids.

Characterization of the MH lla peptide-binding region (PBR) Exon 2 of $\mathrm{MH}$ class II $\alpha$ was also sequenced in a population of 101 seahorse individuals for which the peptide-binding region of the $\mathrm{MH}$ class II $\beta$ locus had previously been characterized [11] to obtain an estimate of population-level variability of this region. PCR amplification and sequencing conditions were identical to those outlined above.

\section{Sequence processing}

All PCR reactions were sequenced in both directions, aligned using ClustalW [33] and visualized in BioEdit v.7.0.9 [34]. Heterozygous sites were coded using IUPAC nomenclature for degenerate positions, and allelic sequences were inferred using the default settings of PHASE V2.1.1 [35]. Individuals for which allelic phase could not be reliably inferred by statistical inference (Phase probabilities $\geq 0.95$ ) were re-amplified and cloned (MH II 1 individual, $\mathrm{MH}$ II $\beta$ : 1 individual, $\mathrm{MH}$ II $\alpha / \mathrm{MH}$ II $\beta$ : 4 individuals). Four to five colonies were sequenced from each cloned individual, allowing the direct determination of individual alleles. All private alleles were separately reamplified and sequenced to verify their identity (MH IIa: 6 individuals, $\mathrm{MH}$ II $\beta$ : 2 individuals, $\mathrm{MH}$ II $\alpha / \mathrm{MH}$ II $\beta$ : 13 individuals).

\section{Analyses of sequence polymorphism/linkage}

Nucleotide diversity $(\pi)$ at MH II $\alpha$ was estimated under the maximum composite likelihood model implemented in Mega v6.0 [36], with standard error estimates derived from 500 bootstrap replicates. Exact tests of HardyWeinberg equilibrium (1,000,000 step Markov Chain, 100,000 dememorization steps) were performed in Arlequin v3.5.1.2 [37].

Analysis of gametic phase of MH II $\alpha$ and II $\beta$ genotypes was performed using the Bayesian ELB approach [38] implemented in Arlequin v.3.5.12. Pairwise linkage 
analysis of unphased MH II $\alpha$ and II $\beta$ data was also carried out using Arlequin (20,000 permutations, 5 EM replicates).

\section{Site-specific tests of positive selection}

Characterization of synonymous and non-synonymous substitutions across the peptide binding region of $\mathrm{MH}$ II $\alpha$ was performed in Mega v6.0 [36] using the Nei-Gojobori method with Jukes-Cantor distances. A one-tailed Z-test of positive selection (500 bootstrap replicates) tested the null hypothesis of neutral evolution for putative peptide binding sites, non-binding sites, and the full peptide binding region.

A neighbor-joining tree was constructed from MH II $\alpha$ alleles using the maximum composite likelihood method implemented in Mega v6.0 [36]. This tree served as a starting tree for a site-specific analysis of positive selection in Codeml v4.8 [39], which compared the fit of a neutral evolution model with recombination (M7) with one allowing for positive selection (M8), using a likelihood-ratio test (LRT). Sites experiencing positive selection were identified following a Bayes Empirical Bayes analysis (posterior probability $\geq 0.95$ ) [40].

\section{Network construction}

An allelic network was constructed to visualize genetic relationships among alleles of the MH II $\alpha$ PBR using TCS v.1.21 [41], and prepared for publication using yED v3.12.2 [42]. A second network was constructed using phased MH II $\alpha$ and II $\beta$ data, in order to visualize the frequency and distribution of $\mathrm{MH}$ class II composite genotypes.

\section{Recombination}

The presence of recombination in the seahorse $\mathrm{MH}$ class II region was investigated using RECCO v.0.93 [43] (10,000 permutations), using a minimum mutation savings criterion of 5 to identify recombinants. Recombination analyses were carried out independently for the $\mathrm{MH}$ II $\alpha$ PBR dataset, and for a concatenated alignment of phased $\mathrm{MH}$ II $\alpha / \mathrm{II} \beta$ data, allowing the identification of intra- and interlocus recombination. Inferred recombination breakpoints for interlocus recombinants included an offset of 4,630 bp of unknown sequence separating the PBRs of the two genes (Figure 1), reflecting uncertainty in the location of breakpoints in the unsequenced region between the two PBRs.

\section{Protein structure}

The quaternary structure of the $\mathrm{MH}$ class II complex of the seahorse was reconstructed via homology modeling of the full-length $\mathrm{MH}$ class II $\alpha$ and II $\beta$ loci to the previously determined crystallographic structure of the mouse $\mathrm{MH}$ class II molecule, using Protinfo PPC [44]. Inferred protein surface models of target and database sequences were annotated and visualized in Chimera v1.6.2 [45].

Protinfo PPC returned five significant hits (structure confidence: 41-55\%), all of which matched PDB models for the extracellular domain of the $\mathrm{MH}$ class II complex of Mus musculus. One of the three top hits (PDB ID: 1ES0: structure confidence 55\%) was selected as a model for the seahorse MH class II complex. The expression vector, peptide and linker of the mouse structure were omitted from the modeled data, as well as 8 aa of $\mathrm{MH}$ II $\beta$ not resolved in the original model, resulting in a total of 182 aa and 180 aa for the MH class II $\alpha$ and II $\beta$ loci, respectively. Known peptide-binding sites for the human $\mathrm{MH}$ class II molecule [3] were annotated on the mouse model, along with sites under positive selection in the seahorse.

\section{Availability of supporting data section}

Sequence data generated for this project have been deposited in GenBank (Accession \#: KP259890-KP259909).

\section{Competing interests}

The authors declare that they have no competing interests.

\section{Authors' contributions}

ABW designed the study, carried out the laboratory and statistical analyses and drafted the manuscript. CMW and $A B$ contributed to the laboratory analyses and participated in the preparation of the manuscript. All authors read and approved the final manuscript.

\section{Acknowledgements}

We thank Annemarie Honegger for discussions concerning homology modelling of the seahorse MHC. This research was supported with funding from the Swiss National Science Foundation, the University of Zurich and City University of New York.

\section{Author details}

'Institute of Evolutionary Biology and Environmental Studies, University of Zurich, Winterthurerstrasse 190, 8057 Zurich, Switzerland. ${ }^{2}$ Department of Biology, Brooklyn College and The Graduate Center, City University of New York, 2900 Bedford Avenue, Brooklyn, New York 11210, USA. ${ }^{3}$ School of Biological Sciences, University of Sydney, Heydon-Laurence Building A08, Sydney, NSW 2006, Australia. ${ }^{4}$ Institute of Medical Molecular Genetics, University of Zurich, Wagistrasse 12, 8952 Schlieren, Switzerland. ${ }^{5}$ Department of Biology, Brooklyn College, 2900 Bedford Avenue, Brooklyn, New York 11210, USA.

Received: 14 August 2014 Accepted: 12 December 2014

Published online: 20 December 2014

\section{References}

1. Kelley J, Walter L, Trowsdale J: Comparative genomics of major histocompatibility complexes. Immunogenetics 2005, 56:683-695.

2. Brown JH, Jardetzky TS, Gorga JC, Stern LJ, Urban RG, Strominger JL, Wiley DC: Three-dimensional structure of the human class-II histocompatibility antigen HLA-DR1. Nature 1993, 364:33-39.

3. Reche PA, Reinherz EL: Sequence variability analysis of human class I and class II MHC molecules: functional and structural correlates of amino acid polymorphisms. J Mol Biol 2003, 331:623-641.

4. Hughes AL: Looking for Darwin in all the wrong places: the misguided quest for positive selection at the nucleotide sequence level. Heredity 2007, 99:364-373.

5. Yeager M, Hughes AL: Evolution of the mammalian MHC: natural selection, recombination, and convergent evolution. Immunol Rev 1999, $167: 45-58$. 
6. Robinson J, Mistry K, McWilliam H, Lopez R, Parham P, Marsh SGE: The IMGT/HLA database. Nucl Acids Res 2011, 39(Database):D1171-D1176.

7. Flajnik MF, Kasahara M: Comparative genomics of the MHC: glimpses into the evolution of the adaptive immune system. Immunity 2001, 15:351-362.

8. Wegner KM: Historical and contemporary selection of teleost MHC genes: did we leave the past behind? J Fish Biol 2008, 73:2110-2132.

9. Gomez D, Conejeros P, Marshall SH, Consuegra S: MHC evolution in three salmonid species: a comparison between class II alpha and beta genes. Immunogenetics 2010, 62:531-542.

10. Stolting KN, Wilson AB: Male pregnancy in seahorses and pipefish: beyond the mammalian model. BioEssays 2007, 29:884-896.

11. Bahr A, Wilson AB: The impact of sex-role reversal on the diversity of the major histocompatibility complex: insights from the seahorse (Hippocampus abdominalis). BMC Evol Biol 2011, 11:121.

12. Bahr A, Wilson AB: The evolution of MHC diversity: evidence of intralocus gene conversion and recombination in a single-locus system. Gene 2012, 497:52-57.

13. Bahr A, Sommer S, Mattle B, Wilson AB: Mutual mate choice in the potbellied seahorse (Hippocampus abdominalis). Behav Ecol 2012, 23:869-878.

14. Haase D, Roth O, Kalbe M, Schmiedeskamp G, Scharsack JP, Rosenstiel P, Reusch TBH: Absence of major histocompatibility complex class II mediated immunity in pipefish, Syngnathus typhle: evidence from deep transcriptome sequencing. Biol Lett 2013, 9:20130044-20130044.

15. Wilson AB, Orr JW: The evolutionary origins of Syngnathidae: pipefishes and seahorses. J Fish Biol 2011, 78:1603-1623.

16. Wilson $A B$, Ahnesjö I, Vincent $A C J$, Meyer $A$ : The dynamics of male brooding, mating patterns, and sex roles in pipefishes and seahorses (family Syngnathidae). Evolution 2003, 57:1374-1386.

17. Ejsmond MJ, Radwan J, Wilson AB: Sexual selection and the evolutionary dynamics of the major histocompatibility complex. Proc R Soc Biol 2014, 281:20141662.

18. McBeth C, Seamons A, Pizarro JC, Fleishman SJ, Baker D, Kortemme T, Goverman JM, Strong RK: A new twist in TCR diversity revealed by a forbidden aß TCR. J Mol Biol 2008, 375:1306-1319.

19. Jeffreys AJ, Kauppi L, Neumann R: Intensely punctate meiotic recombination in the class II region of the major histocompatibility complex. Nat Genet 2001, 29:217-222

20. Yauk $C L$, Bois $P R$, Jeffreys $A J$ : High-resolution sperm typing of meiotic recombination in the mouse MHC Eß gene. EMBO J 2003, 22:1389-1397.

21. Jeffreys AJ, May CA: Intense and highly localized gene conversion activity in human meiotic crossover hot spots. Nat Genet 2004, 36:151-156.

22. Van Look KJW, Dzyuba B, Cliffe A, Koldewey HJ, Holt WV: Dimorphic sperm and the unlikely route to fertilisation in the yellow seahorse. J Exp Biol 2007, 210:432-437.

23. Čížková D, de Bellocq JG, Baird SJE, Piálek J, Bryja J: Genetic structure and contrasting selection pattern at two major histocompatibility complex genes in wild house mouse populations. Heredity 2011, 106:727-740.

24. Miyasaka T, Takeshima S, Matsumoto Y, Kobayashi N, Matsuhashi T, Miyazaki $Y$, Tanabe $Y$, Ishibashi $K$, Sentsui $H$, Aida $Y$ : The diversity of bovine MHC class II DRB3 and DQA1 alleles in different herds of Japanese Black and Holstein cattle in Japan. Gene 2011, 472:42-49.

25. Babik W: Methods for MHC genotyping in non-model vertebrates. Mol Ecol Resour 2010, 10:237-251.

26. Sommer S, Courtiol A, Mazzoni CJ: MHC genotyping of non-model organisms using next-generation sequencing: a new methodology to deal with artefacts and allelic dropout. BMC Genomics 2013, 14:542.

27. Lighten J, van Oosterhout C, Bentzen P: Critical review of NGS analyses for de novo genotyping multigene families. Mol Ecol 2014:in press.

28. Huchard E, Albrecht C, Schliehe-Diecks S, Baniel A, Roos C, Kappeler PM, Peter PMK, Brameier M: Large-scale MHC class II genotyping of a wild lemur population by next generation sequencing. Immunogenetics 2012, 64:895-913.

29. Pavey SA, Sevellec M, Adam W, Normandeau E, Lamaze FC, Gagnaire P-A, Filteau M, Hebert FO, Maaroufi H, Bernatchez L: Nonparallelism in MHCII diversity accompanies nonparallelism in pathogen infection of lake whitefish (Coregonus clupeaformis) species pairs as revealed by next-generation sequencing. Mol Ecol 2013, 22:3833-3849.
30. Radwan J, Zagalska-Neubauer M, Cichoń M, Sendecka J, Kulma K, Gustafsson L, Babik W: MHC diversity, malaria and lifetime reproductive success in collared flycatchers. Mol Ecol 2012, 21:2469-2479.

31. Wilson AB, Martin-Smith KM: Genetic monogamy despite social promiscuity in the pot-bellied seahorse (Hippocampus abdominalis). Mol Ecol 2007, 16:2345-2352.

32. Sommer $\mathrm{S}$, Whittington $\mathrm{CM}$, Wilson $\mathrm{AB}$ : Standardised classification of pre-release development in male-brooding pipefish, seahorses, and seadragons (Family Syngnathidae). BMC Dev Biol 2012, 12:39.

33. Thompson JD, Higgins DG, Gibson TJ: CLUSTAL W: improving the sensitivity of progressive multiple sequence alignment through sequence weighting, position-specific gap penalties and weight matrix choice. Nucl Acids Res 1994, 22:4673-4680.

34. Hall TA: BioEdit: a user-friendly biological sequence alignment editor and analysis program for Windows 95/98/NT. Nucl Acids Symp 1999, 41:95-98.

35. Stephens M, Donnelly P: A comparison of Bayesian methods for haplotype reconstruction from population genotype data. Am J Hum Genet 2003, 73:1162-1169.

36. Tamura K, Stecher G, Peterson D, Filipski A, Kumar S: MEGA6: molecular evolutionary genetics analysis version 6.0. Mol Biol Evol 2013, 30:2725-2729.

37. Excoffier $L$, Lischer HEL: Arlequin suite ver 3.5: a new series of programs to perform population genetics analyses under Linux and Windows. Mol Ecol Resour 2010, 10:564-567.

38. Excoffier L, Laval G, Balding D: Gametic phase estimation over large genomic regions using an adaptive window approach. Hum Genomics 2003, 1:7-19.

39. Yang Z: PAML 4: phylogenetic analysis by maximum likelihood. Mol Biol Evol 2007, 24:1586-1591.

40. Yang Z, Wong WSW, Nielsen R: Bayes empirical bayes inference of amino acid sites under positive selection. Mol Biol Evol 2005, 22:1107-1118.

41. Clement M, Posada D, Crandall KA: TCS: a computer program to estimate gene genealogies. Mol Ecol 2000, 9:1657-1659.

42. yWorks: yED Graph Editor. Tuebingen, Germany: yWorks; 2009.

43. Maydt J, Lengauer T: Recco: recombination analysis using cost optimization. Bioinformatics 2006, 22:1064-1071.

44. Kittichotirat W, Guerquin M, Bumgarner RE, Samudrala R: Protinfo PPC: a web server for atomic level prediction of protein complexes. Nucl Acids Res 2009, 37(Web Server):W519-W525.

45. Pettersen EF, Goddard TD, Huang CC, Couch GS, Greenblatt DM, Meng EC, Ferrin TE: UCSF Chimera-a visualization system for exploratory research and analysis. J Comp Chem 2004, 25:1605-1612.

\section{Submit your next manuscript to BioMed Central and take full advantage of:}

- Convenient online submission

- Thorough peer review

- No space constraints or color figure charges

- Immediate publication on acceptance

- Inclusion in PubMed, CAS, Scopus and Google Scholar

- Research which is freely available for redistribution 